OPEN ACCESS

Edited by:

Manuel Espinosa,

Spanish National Research

Council - Centre for Biological

Research, Spain

Reviewed by:

Kurt Henry Piepenbrink,

The University of Maryland School of

Medicine, USA

Jan Löwe,

Medical Research Council, UK

*Correspondence:

María A. Oliva

marian@cib.csic.es

Specialty section:

This article was submitted to

Molecular Recognition,

a section of the journal

Frontiers in Molecular Biosciences

Received: 28 June 2016

Accepted: 24 August 2016

Published: 09 September 2016

Citation:

Oliva MA (2016) Segrosome Complex Formation during DNA Trafficking in Bacterial Cell Division.

Front. Mol. Biosci. 3:51. doi: 10.3389/fmolb.2016.00051

\section{Segrosome Complex Formation during DNA Trafficking in Bacterial Cell Division}

\author{
María A. Oliva * \\ Department of Chemical and Physical Biology, Centro de Investigaciones Biológicas, Consejo Superior de Investigaciones \\ Científicas, Madrid, Spain
}

Bacterial extrachromosomal DNAs often contribute to virulence in pathogenic organisms or facilitate adaptation to particular environments. The transmission of genetic information from one generation to the next requires sufficient partitioning of DNA molecules to ensure that at least one copy reaches each side of the division plane and is inherited by the daughter cells. Segregation of the bacterial chromosome occurs during or after replication and probably involves a strategy in which several protein complexes participate to modify the folding pattern and distribution first of the origin domain and then of the rest of the chromosome. Low-copy number plasmids rely on specialized partitioning systems, which in some cases use a mechanism that show striking similarity to eukaryotic DNA segregation. Overall, there have been multiple systems implicated in the dynamic transport of DNA cargo to a new cellular position during the cell cycle but most seem to share a common initial DNA partitioning step, involving the formation of a nucleoprotein complex called the segrosome. The particular features and complex topologies of individual segrosomes depend on both the nature of the DNA binding protein involved and on the recognized centromeric DNA sequence, both of which vary across systems. The combination of in vivo and in vitro approaches, with structural biology has significantly furthered our understanding of the mechanisms underlying DNA trafficking in bacteria. Here, I discuss recent advances and the molecular details of the DNA segregation machinery, focusing on the formation of the segrosome complex.

\footnotetext{
Keywords: DNA segregation, partitioning systems, segrosome, partitioning complex, nucleoprotein complex, ParB, ParR, TubR
}

\section{DNA MAINTENANCE DURING BACTERIAL CELL DIVISION}

The process of DNA segregation is a crucial stage of the bacterial cell cycle and it depends on the precise coordination with other cellular events. The faithful inheritance of genetic information during cell division ensures that each daughter cell receives a copy of the newly replicated DNA. In many organisms, the DNA-encoded genome consists of a core genome (the chromosome) and accessory genomes (extra-chromosomal, mobile genetic elements, MGEs). MGEs (plasmids, phages, conjugative transposons, etc.) often confer evolutionary advantages to the host bacteria, including the adaptation to different environmental niches. Many, if not most, naturally occurring MGEs are in low or unique copy number and thus bring their own post-replication survival apparatus encoded in stability determinants (partitioning systems, toxin-antitoxin systems, and multimer-resolution systems). 
Partitioning (par) systems help to reliably segregate sister DNAs via a process that could be seen as functionally analogous to the mitotic segregation of chromosomes in eukaryotic cells. The best studied and probably the most common partitioning systems constitute a compact genetic module that is tightly autoregulated by one of the gene products and consists of only three elements: a cis-acting DNA sequence and two trans-acting proteins. The DNA sequence denotes a par site or centromerelike region, and can be located at a single site (upstream or downstream of the operon) or at multiple positions within the MGE. The trans-acting proteins consist of a centromerebinding protein (CBP) that binds to the centromere and forms a nucleoprotein complex (partition complex or segrosome), and a motor protein (an NTPase), that sometimes is a cytomotive filament, which effectively moves the MGE inside the bacteria through direct interaction with the segrosome. Initially, these systems were classified as follows, based on the molecular nature of the NTPase: type I (Walker A-type ATPase), which further divided into Ia and Ib based on differences in the trans-acting proteins and the position of the centromere in the operon; and type II (cytomotive, actin-like ATPase) (Gerdes et al., 2000). Recently, an additional type III system has emerged, in which a cytomotive, tubulin-like GTPase serves as the motor protein (Larsen et al., 2007). Further, there may exist a type IV partitioning system, in which only a cis-acting DNA site and a DNA binding protein seem required for plasmid maintenance (Simpson et al., 2003; Guynet and de la Cruz, 2011). Hence, they may use a host bacteria's motor protein to track the DNA, or may even segregate passively by establishing an association with the chromosome (Guynet and de la Cruz, 2011).

It seems that partitioning systems share a common initial step that involves the specific recognition of the centromeric DNA region by the CBP. This step is crucial for assembly of the segrosome and subsequent events during DNA segregation. However, there is a considerable divergence among par sites and CBPs display different domain folds and organization (Hayes and Barilla, 2006; Baxter and Funnell, 2014), indicating differences in the segrosome assembly process and by extension, the corresponding partitioning mechanism. Here, I review the molecular mechanisms underlying segrosome formation in the partitioning systems that have been studied, focusing on those where structural information is available. Despite these variations in centromere sequences and the natures of the CBPs, common to all systems is the formation of the nucleoprotein complex that I propose may be categorized into two classes: those that mediate DNA segregation via bridging and those that do so via wrapping.

\section{DNA BRIDGING IN TYPE IA PARTITIONING SYSTEMS}

Many plasmids, phages and chromosomes encode type Ia partitioning systems (Martin et al., 1987; Balzer et al., 1992; Lewis and Errington, 1997; Grigoriev and Lobocka, 2001). No single, common segrosome assembly mechanism has been described for these systems, probably owing to the wide diversity of centromeres and variations on the CBPs (below). The exact nature of the partition complex is unknown, if it even exists in only one particular conformation, but the CBP bridges distant regions of DNA via both specific and non-specific binding (Rodionov et al., 1999; Bingle et al., 2005; Murray et al., 2006; Schumacher et al., 2007b; Graham et al., 2014), enabling the formation of a nucleoprotein complex linking and/or spanning thousands of base pairs with a small number of CBPs. Furthermore, spreading of the CBP has a masking effect on the function of the covered DNA, preventing interaction between the motor protein and the DNA and favoring the interaction with the segrosome (Bouet et al., 2007).

While the sequences of cis-acting sites (parS, sopC or $\mathrm{O}_{\mathrm{B}}$ ) vary, the sites always contain inverted repeats. The parS site contains two different repeats asymmetrically arranged around a binding site for the IHF protein (Davis and Austin, 1988; Funnell, 1988b). One of the motifs is a heptamer (A-box) and the other a hexamer (B-box). Binding of IHF bends the DNA by $180^{\circ}$, thus strongly promoting ParB binding (Funnell, 1988a; Funnell and Gagnier, 1993; Rice et al., 1996; Bouet et al., 2000; Surtees and Funnell, 2001). Some chromosomes contain several parS sites dispersed over $\sim 15 \%$ of the DNA, surrounding the replication origin (Lin and Grossman, 1998; Livny et al., 2007). However, the chromosomal parS sites consist exclusively of palindromic A-box motifs. SopC and $\mathrm{O}_{\mathrm{B}}$ sites comprise only one type of short inverted repeats contained within longer iterons that can be found either at a single locus (Mori et al., 1986) or scattered across the genome (Balzer et al., 1992; Ravin and Lane, 1999). The function of the regions flanking the inverted repeats is puzzling, as their presence is not conserved (Ravin and Lane, 1999). Similarly, the need for more than one iteron remains unclear, as in almost all cases a single copy is sufficient for segregation (Martin et al., 1987; Williams et al., 1998; Yates et al., 1999). However, given that the full-length centromere maximizes partitioning efficiency (Martin et al., 1987), the architecture of each segrosome may reflect evolutionary pressure on how well the systems work.

Type I CBPs are members of the ParB protein superfamily but show low sequence conservation. ParB, Spo0J, SopB, and KorB share the same domain organization, consisting of three flexibly linked domains (Schumacher et al., 2010): N-terminal, central (with a DNA-binding helix-turn-helix, HTH, motif), and C-terminal domains, which have been seen in various inter-domain conformations (Chen et al., 2015). The central domain is responsible for the primary CBP-DNA interaction, and the $\mathrm{N}$ - and C-terminal domains contribute to CBPs spreading around the centromere DNA. ParB proteins show high structural conservation only in the central domain, probably due to the presence of the HTH motif. For DNA binding, the HTH recognition helix inserts into the major groove, but there are differences between CBPs. In ParB, the HTH motif binds the pars box-A exclusively via the recognition helix (Schumacher and Funnell, 2005). SopB uses the recognition helix and an Arg outside the HTH (Schumacher et al., 2010; Sanchez et al., 2013). Spo0J binding is very similar to that observed for SopB but uses a Lys instead of an Arg and form additional specific contacts via another Arg and a Glu (Chen et al., 2015). Surprisingly, the HTH motif of KorB mediates only non-specific interactions, and DNA 
binding specificity depends on contacts formed via a Thr and an Arg located outside the HTH (Khare et al., 2004). All these proteins bind DNA as dimers, whereby each molecule generally interacts with opposite sides of the inverted repeat (Figure 1A). However, in the crystal structure of ParB, the monomers of each dimer bind to box elements of different DNA molecules, suggesting a possible DNA crosslinking function or, that crystal packing occluded correct binding (Schumacher and Funnell, 2005).

ParBs' flexible N-terminal domain is responsible for the binding to the motor protein, oligomerization of the CBP around the centromere, and also loading of bacterial condensin (Gruber and Errington, 2009; Sullivan et al., 2009; Minnen et al., 2011; Havey et al., 2012; Graham et al., 2014). Figure 1B shows the domain topology of Spo0J ( $\alpha 1-\beta 1-\beta 2-\alpha 2-\beta 3-\alpha 3)$, in which $\beta$-strands fold to form a $\beta$-sheet (Leonard et al., 2004). The two conserved motifs, box 1 and box 2 [with an "arginine patch," (Yamaichi and Niki, 2000)] are located between $\alpha 1$ and $\beta 1$ and between $\beta 2$ and $\alpha 2$, respectively (Chen et al., 2015). Upon DNA binding the protein opens into an elongated, $78 \AA$ long structure, leaving the $\mathrm{N}$-terminal domain exposed and available for proteinprotein interactions (Chen et al., 2015). These interactions are

A

Central domain: DNA specific binding

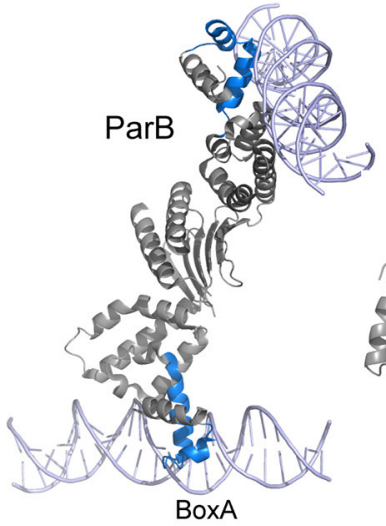

HTH specific binding

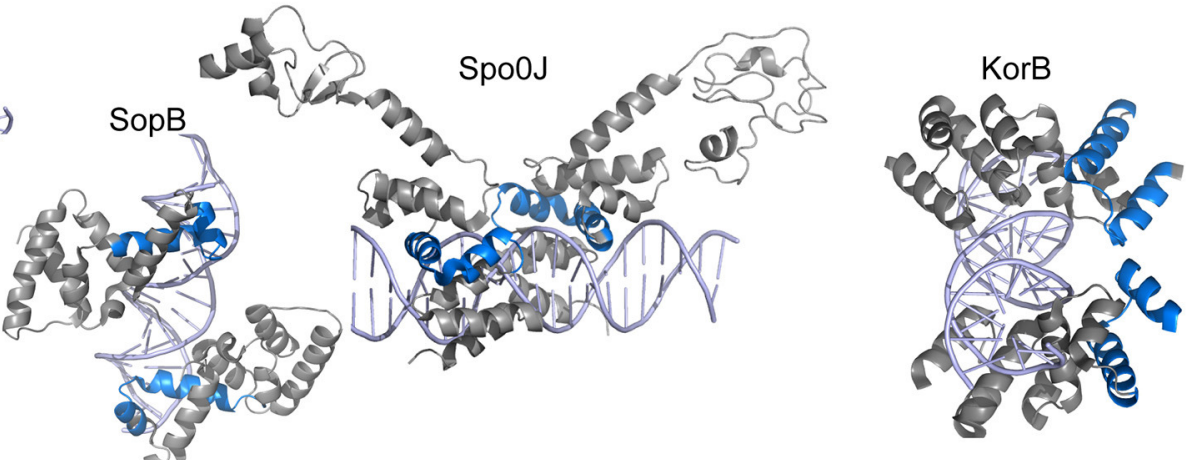

HTH non-specific binding
B N-terminal domain: protein-protein contacts

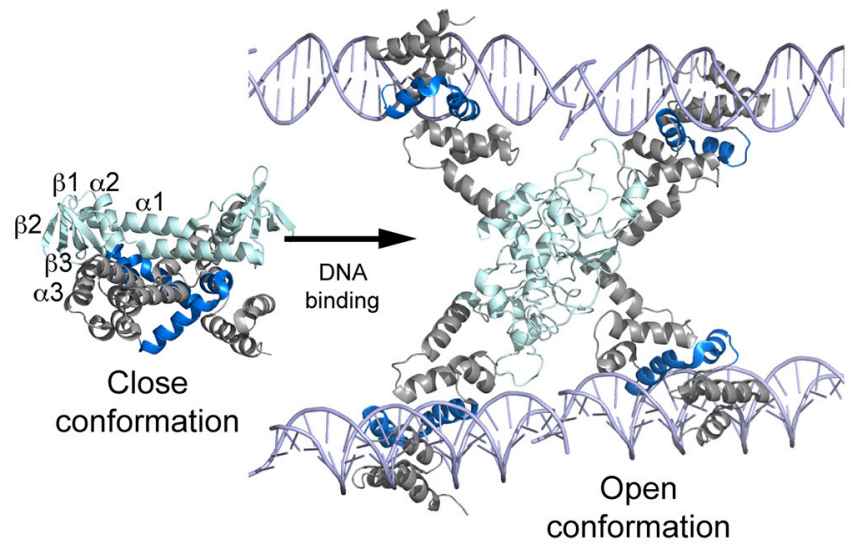

DNA crosslinking
C C-terminal domain: dimerization \& DNA contact

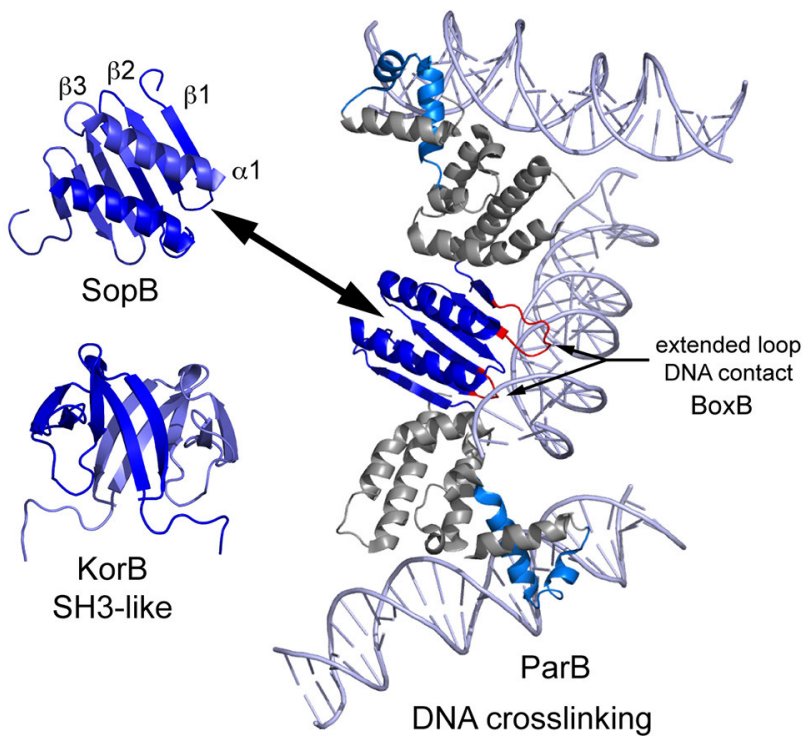

FIGURE 1 | Scheme showing the structures of Type la CBPs domains and their interaction with DNA during bridging. (A) Central domain showing primary specific DNA interaction of ParB, SopB, Spo0J, and KorB. These proteins bind as dimers, making contact with both sides of the DNA. However, ParB dimerization generate a different contact mechanism that involves the bridging of different DNA molecules. (B) SpoOJ N-terminal domain structures, both unbound and DNA-bound. Binding to the centromere induces a domain rearrangement that favors DNA bridging. (C) The C-terminal domain folding differs considerably between ParB/sopB and KorB. ParB folding includes extended loops that make contacts with DNA, favoring bridging distant molecules. DNA is shown in light purple, the HTH motif in blue, the $\mathrm{N}$-terminal domain in light blue, and the $\mathrm{C}$-terminal domain in dark blue. 
very flexible, but always include box 1 and 2 (Kusiak et al., 2011). Through this arrangement, the N-terminal domain is able to assist CBP spreading (Kusiak et al., 2011; Graham et al., 2014). Due to a lack of structural data, it remains unclear how the flexibility of the domains and their binding to DNA enables simultaneous or alternative interactions with the condensin and motor proteins.

The C-terminal domain is the most divergent, but in all these proteins shares the ability to dimerize (Leonard et al., 2004; Chen et al., 2015). The domain topology of ParB/SopB is $\beta 1-\beta 2-\beta 3-\alpha 1$, where the $\beta 3 \mathrm{~s}$ of each monomer combine to form a continuous 6 -stranded $\beta$-sheet and the helices interact to form an antiparallel coiled-coil (Figure 1C, Schumacher and Funnell, 2005; Schumacher et al., 2010). ParB contains extended loops between $\beta 1-\beta 2$ and $\beta 2-\beta 3$ that form highly specific contacts with the parS B-box (Schumacher and Funnell, 2005), generating a secondary DNA binding domain and contributing to DNA bridging during segrosome formation. By contrast, the C-terminal domain of KorB displays a completely different folding pattern, resembling an $\mathrm{SH} 3$ protein and consisting of a 5 -stranded antiparallel $\beta$ sheet (Delbruck et al., 2002). However, crosslinking studies suggest that this domain also facilitates DNA binding (Delbruck et al., 2002).

\section{SEGROSOME ASSEMBLY VIA WRAPPING}

This strategy involves the formation of a filamentous nucleoprotein complex, in which the CBP wraps the centromere (type Ia partition systems) or the DNA wraps around a CBP oligomer (type II and III partition systems). The resulting segrosome is a single and discrete structure.

\section{Type Ib Systems}

Surprisingly, the arrangement of the components in Type Ib systems is the only common aspect shared with the aforementioned systems. The interactions between their main components are different, and so may be the segregation mechanism. The centromere site localizes upstream of the par operon and consists of direct and inverted repeats. However, in plasmid pCXC100 the centromeric site contains only direct repeats (Yin et al., 2006; Huang et al., 2011). The CBPs, which also functions as repressors (Carmelo et al., 2005; Weihofen et al., 2006) are small proteins that share the arrangement into $\mathrm{N}$ - and C-terminal domains (Figure 2C). The N-terminal domain, which shows a highly divergent sequence, is flexible and unstructured, and includes a conserved arginine finger that has been implicated in the activation of ATP hydrolysis in the motor protein (Barilla et al., 2007). The C-terminal domain topology is $\beta 1-\alpha 1-\alpha 2$ and includes a ribbon-helix-helix (RHH) DNA-binding motif (Murayama et al., 2001; Golovanov et al., 2003; Huang et al., 2011). The $\beta 1$ strand from two different molecules pairs into an antiparallel $\beta$-ribbon, meaning that these CBPs are also present as dimers in solution (Barilla and Hayes, 2003; Golovanov et al., 2003).

Plasmid pSM19035 harbors a unique partitioning system: rather than being encoded in a single operon, each gene is transcribed separately from different promoters. The full centromere contains 3 separate parS sites, consisting of 9, 7 , and 10 iterons that occur twice in the plasmid genome (parS1, parS1', parS2, parS2', parS3, parS3', de la $\mathrm{Hoz}$ et al., 2000, 2004; Dmowski et al., 2006). However, parS2 appears to be the main centromeric sequence (Dmowski and KernZdanowicz, 2016). Interestingly, each parS overlaps with the promoters of genes involved in plasmid copy number and maintenance: parS1 with $\mathrm{P} \delta$, parS2 with $\mathrm{P} \omega$ and parS3 with $\mathrm{P}_{\text {cops }}$ (de la Hoz et al., 2000). The CBP, $\omega$, binds to each parS with different affinities, depending on the number of iterons (de la Hoz et al., 2004). This feature may be crucial to finetune repressor affinity for different promoters (Weihofen et al., 2006). The nucleoprotein complex is a left-handed protein helix that wraps the DNA (Weihofen et al., 2006) covering only the parS site (Pratto et al., 2009). Protein binding to both direct and inverted repeats involves comparable interactions, due to the pseudo-symmetry of the dimer (Weihofen et al., 2006, Figure 2C). Binding induces minor structural changes mainly affecting the loop connecting $\alpha 1$ and $\alpha 2$. In contrast to other RHH DNA-binding proteins, there is no DNA bending (Pratto et al., 2009). Because the DNA is not curved, $\omega$ first makes contact with the DNA major groove via base specific interactions with residues on the $\beta$-sheet and then the $\mathrm{N}$ termini of the $\alpha 2$ helices clamp the phosphate backbones (Weihofen et al., 2006). Assuming nearly straight DNA, the number and orientation of repetitions will affect the distances between helices $\alpha 1$ of adjacent $\omega$ dimers, thereby modulating the cooperativity. The motor protein, $\delta$, binds non-specifically to DNA but is recruited to the location of the segrosome (Pratto et al., 2009) to form a ternary complex, giving rise to intermolecular pairing of parS regions (Pratto et al., 2008, 2009). This bridging may increase the local concentration of $\omega$, in turn increasing the ATPase activity of the motor protein and thus inducing detachment of this protein and promoting mobility (Pratto et al., 2009). This system may combines both DNA wrapping mechanisms (during segrosome formation) and DNA bridging mechanisms (when the motor protein participates in segregation).

In plasmid TP228, the centromere (parH) is continuous and consists of direct and inverted repeats separated by AT-rich regions. A DNA region between the operon genes and the centromere $\left(\mathrm{O}_{\mathrm{F}}\right)$ comprises more repeats that play important roles in partitioning and transcription regulation (Zampini et al., 2009; Wu et al., 2011). Binding of the CBP, ParG, to parH occurs via the RHH motif, but unlike in $\omega$, ParG is also dependent on the protein's N-terminal tail, which modulates binding affinity (Golovanov et al., 2003). Apparently, the AT-enriched spacers may increase the binding cooperativity of ParG to DNA (Wu et al., 2011). Like plasmid pSM19035, the centromere site is not curved and ParG binding does not induce DNA bending. The motor protein, ParF polymerizes into filaments and does not bind to DNA (Barilla et al., 2005; Schumacher et al., 2012). The N-terminal domain of ParG is not only important for the activation of the ATPase but also facilitates ParF filament nucleation and bundling (Barilla et al., 2007). Furthermore, in contrast to all other described systems, the ParF-ParG interaction is not dependent on the formation of the segrosome. This 


\section{A Type II systems}

ParR dimer

N-terminal<smiles>CCCCCC(CCCC)(CCCCC)c1ccccc1</smiles>

C-terminal

B Type III systems
TubR dimer

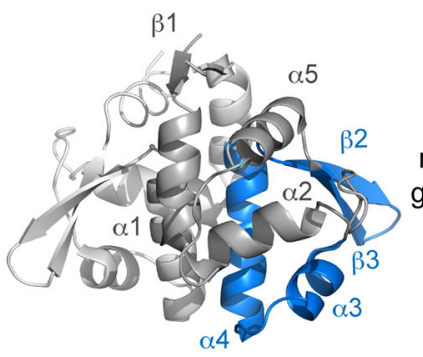

recognition helix
ParR dimer-of-dimers

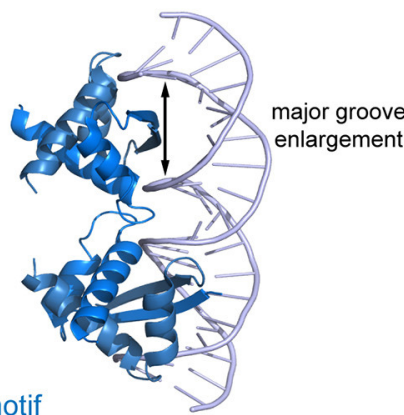

Super-helical complex

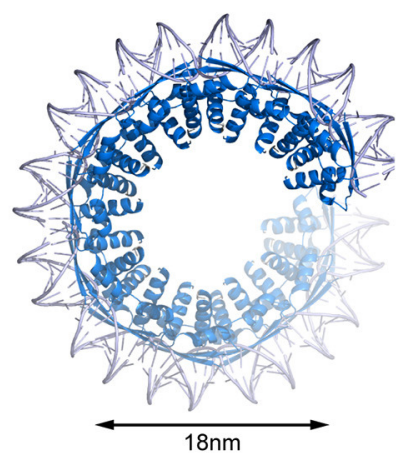

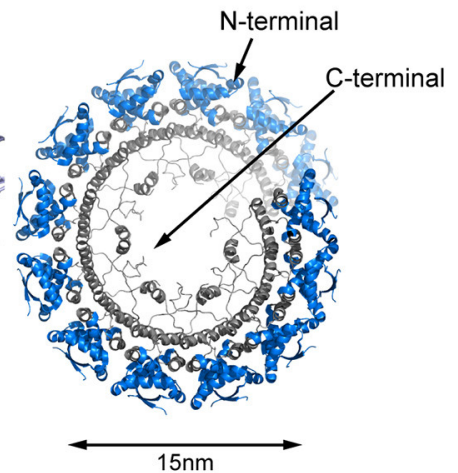

Filamentous vs. Helical complex

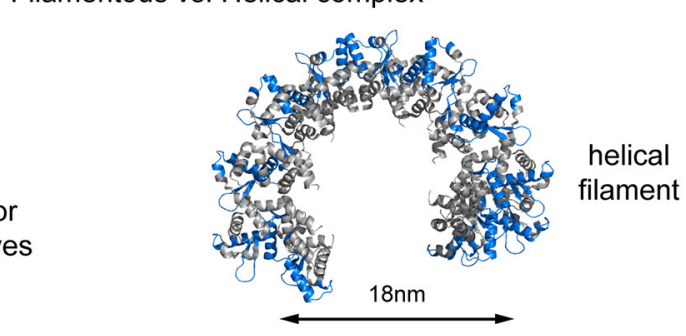

HTH motif
TubR DNA binding

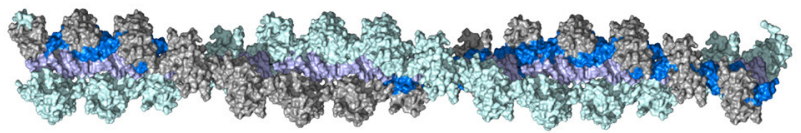

flexible filament

c Type lb systems

$\omega$ dimer

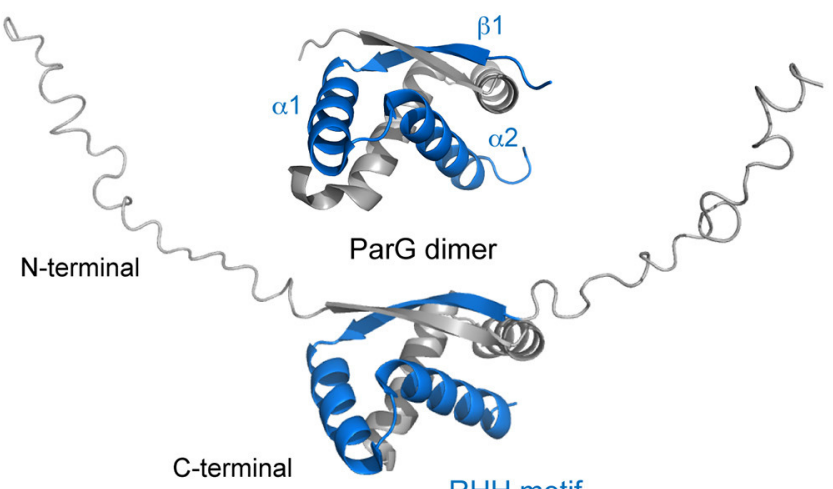

RHH motif $\omega$ DNA binding

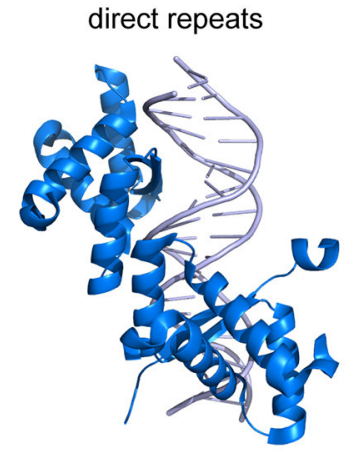

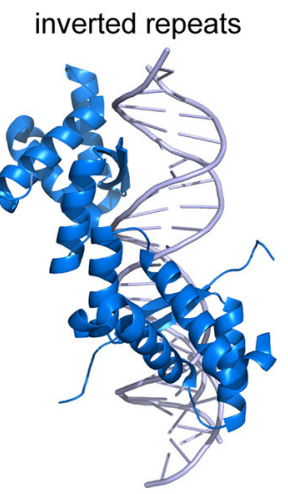

FIGURE 2 | Structural comparison of CBPs involved in segrosome assembly by wrapping. (A) Type I| partition systems. Structure of ParR dimer (left), showing topology and the RHH motif; DNA changes upon ParR binding (middle), with enlargement of the DNA major groove; and formation of the the segrosome complex by DNA wrapping of the ParR super-helical oligomer (right), leaving the ParR C-terminal tail in the helix inside. (B) Type III partition systems. Structure of TubR dimer (left), showing topology and the HTH motif; the TubR-DNA binding mechanism (middle), in which the HTH makes contacts with the DNA major groove and the wing forms contacts with the minor groove; and putative filamentous vs. helical segrosome complexes (right), according to two crystal packing arrangements. (C) Type Ib partition systems. Structures of ParG and $\omega$ dimers (left), showing the RHH motif and the flexible N-terminal domain, and protein binding to direct and inverted repeats in equivalent ways (right). 
suggests that pSM19035 and TP228 despite sharing the same type Ib partitioning system employ distinct segregation mechanisms.

\section{Type II Systems}

The centromeric site ( $p a r C$ ) consists of tandem repeats localized in a single locus upstream of the operon. The arrangement can be continuous (plasmid pSK41, Schumacher et al., 2007a) or split into two regions (plasmid R1), with the par cassette promoter in the middle (Dam and Gerdes, 1994). However, the resulting segregation complexes are very similar. The CBP, ParR, contains two domains, an N-terminal domain with a RHH DNA binding motif (as seen in type Ib systems), and a C-terminal domain that is involved in the interaction with the motor protein. The domain topology of the ParR N-terminal domain is $\beta 1-\alpha 1-\alpha 2-\alpha 3-\alpha 4-\alpha 5$ (Figure 2A). The $\beta 1$-strands from two monomers combine in an antiparallel fashion and the $\alpha 1-\alpha 2$ helices come together to form an extensive dimer (Moller-Jensen et al., 2007; Schumacher et al., 2007a). The C-terminal domain includes a 3-helix cap that reinforces the tight dimerization of the $\mathrm{N}$-terminal domain and an unstructured C-terminal tail with a high degree of sequence conservation (Moller-Jensen et al., 2007).

The nucleoprotein complex forms a discrete helical arrangement with a diameter of 15-nm (Moller-Jensen et al., 2007; Hoischen et al., 2008). The structure of the nucleoprotein complex (Schumacher et al., 2007a) reveals a continuous helical array in the crystal packing (Figure 2A). Each turn consists of 6 symmetrical pairs of dimers (involving the assembly of 12 ParR dimers), producing distinct negative and positive electrostatic on the inner and outer surfaces of the helix. The DNA wraps ParR by interacting with the outer, positively charged surface of the super helix, with each dimer binding one parC iteron. When the centromere is split in two, the promoter region forms a DNA loop that protrudes out of the ParR-parC ring structure (Hoischen et al., 2008; Salje and Lowe, 2008), repressing the promoter (Jensen et al., 1994; Breuner et al., 1996), and regulating transcription of the partition genes (Salje and Lowe, 2008). The DNA is bent by $46^{\circ}$ and widened so that the major groove grows from 11 to $14 \AA$ (Schumacher et al., 2007a). The groove enlargement allows the insertion of the $\mathrm{RHH}$ motif, as described for other DNA-binding $\mathrm{RHH}$ proteins (Somers and Phillips, 1992; Raumann et al., 1994; Gomis-Ruth et al., 1998). Interestingly, the phosphate contacts cluster at the $5^{\prime}$ ends of the $10-\mathrm{bp}$ repeats, creating the closest physical associations between ParR and the DNA. Full-length ParR from plasmid pB171 crystallized in a helical superstructure in the absence of DNA, with a diameter very similar to that measured in the nucleoprotein complex (15 vs. $18 \mathrm{~nm}$ ) (Figure 2A). Moreover, the protein arrangement into dimers and the electrostatic distribution are also similar (Moller-Jensen et al., 2007). These observations lead to the question; which event occurs first? If ParR assembly into a super-helical structure occurs first, then the macromolecular complex may recruit parC. Otherwise, the centromere might function as a scaffold for ParR oligomerization.

For ParR, the segrosome structure positions the conserved C-tails clustered on the inside surface of the helix, where they mediate binding to the motor protein, ParM (Schumacher et al., 2007a; Salje and Lowe, 2008). The ParR tail binds to a hydrophobic pocket in ParM in an interaction resembling that described for actin polymer modulators and the barbed end of actin filaments (Gayathri et al., 2012). Furthermore, the segrosome binds only at the growing end of the polar ParM double helical filament favoring filament growth via a forminlike mechanism (Gayathri et al., 2012). Why does the ParRParM interaction require the clustering of so many ParR tails? It is possible that several tails bind to a single ParM molecule with distinct affinities, regulating ParM filament dynamics. Alternatively, the presence of free tails may be necessary to explore the space around the filament end and to facilitate the addition of ParM molecules to the growing filament while remaining attached at all times.

\section{Type III Systems}

The type III system were the most recently discovered partitioning systems (Larsen et al., 2007). For TubZRC, the centromeric site $(t u b C)$ is localized upstream of the operon and contains several direct repeats in a single locus that can be split into two (pBtoxis) or three (pBsph) blocks, resembling discontinuous parC sites (Aylett and Lowe, 2012; Ge et al., 2014a). During partitioning, the CBP (TubR in this case) mediates the assembly of the segrosome nucleoprotein complex and acts as a repressor of $t u b R Z$ transcription (Tang et al., 2006; Larsen et al., 2007; Ge et al., 2014a). TubR is a small wingedhelix DNA-binding protein with a high degree of structure conservation. The topology is $\beta 1-\alpha 1-\alpha 2-\alpha 3-\alpha 4-\beta 2-\beta 3-\alpha 5$, where the $\alpha 3-\alpha 4$ helices form the HTH motif ( $\alpha 4$ is the "recognition helix") and the loop between $\beta 2-\beta 3$ forms the wing (Figure $2 \mathbf{B}$, $\mathrm{Ni}$ et al., 2010). Interestingly, TubR forms a highly intertwined dimer involving the canonical HTH motif, resulting in an atypical protein-DNA binding (Aylett and Lowe, 2012). The Ntermini of both recognition helices in a dimer protrude into the major groove of the DNA, while the acidic patch in the wing complements the DNA backbone phosphate in the minor groove. The nucleoprotein complex takes the shape of a flexible filament, with TubR wrapping helically around both sides of $t u b C$ (Aylett and Lowe, 2012, Figure 2B). The filamentous complex closes to form 18-nm wide ring-like structures (Aylett and Lowe, 2012). However, the structure of plasmid pBM400 TubR, with no DNA bound, reveals a helical arrangement, resembling the ParR superhelical complex (Figures 2A,B). It thus remains unclear whether the segrosome complex is formed by TubR wrapping of the DNA or by DNA wrapping of the TubR oligomer, which could lead to different interacting mechanism with the motor protein (TubZ).

TubR binds to TubZ C-terminal tail (Ni et al., 2010). However, the interaction is only possible following formation of the filamentous segrosome. Neither TubR alone (Oliva et al., 2012) nor TubR bound to either of the two-iteron clusters are capable of interacting with TubZ (Aylett and Lowe, 2012; Fink and Lowe, 2015). Therefore, the clustering of TubR may generate the binding site for TubZ. Differently to type II systems, the segrosome tracks the shrinking minus end of the TubZ filament, suggesting a pulling segregation mechanism (Fink and Lowe, 2015). 
Type III partition systems involve a third protein with a predicted HTH DNA-binding motif and a long coiled-coil domain (TubY), located downstream of the partition operon (Oliva et al., 2012). TubY seems to be a regulator protein that modulates TubZ assembly (Oliva et al., 2012) and also acts as a transcriptional activator (Ge et al., 2014b) but the exact molecular mechanisms remain elusive.

It is still common for new partitioning systems to be discovered in plasmids, phages, and on chromosomes. Together with a growing body of molecular insights these will help to broaden our understanding of DNA trafficking during bacterial cell division and in particular how DNA is attached to the CBP during segrosome

\section{REFERENCES}

Aylett, C. H., and Lowe, J. (2012). Superstructure of the centromeric complex of TubZRC plasmid partitioning systems. Proc. Natl. Acad. Sci. U.S.A. 109, 16522-16527. doi: 10.1073/pnas.1210899109

Balzer, D., Ziegelin, G., Pansegrau, W., Kruft, V., and Lanka, E. (1992). KorB protein of promiscuous plasmid RP4 recognizes inverted sequence repetitions in regions essential for conjugative plasmid transfer. Nucleic Acids Res. 20, 1851-1858. doi: 10.1093/nar/20.8.1851

Barillà, D., Carmelo, E., and Hayes, F. (2007). The tail of the ParG DNA segregation protein remodels ParF polymers and enhances ATP hydrolysis via an arginine finger-like motif. Proc. Natl. Acad. Sci. U.S.A. 104, 1811-1816. doi: 10.1073/pnas.0607216104

Barillà, D., and Hayes, F. (2003). Architecture of the ParF*ParG protein complex involved in prokaryotic DNA segregation. Mol. Microbiol. 49, 487-499. doi: 10.1046/j.1365-2958.2003.03564.x

Barillà, D., Rosenberg, M. F., Nobbmann, U., and Hayes, F. (2005). Bacterial DNA segregation dynamics mediated by the polymerizing protein ParF. EMBO J. 24, 1453-1464. doi: 10.1038/sj.emboj.7600619

Baxter, J. C., and Funnell, B. E. (2014). Plasmid Partition Mechanisms. Microbiol. Spectr. 2:PLAS-0023-2014. doi: 10.1128/microbiolspec.PLAS-0023-2014

Bingle, L. E., Macartney, D. P., Fantozzi, A., Manzoor, S. E., and Thomas, C. M. (2005). Flexibility in repression and cooperativity by KorB of broad host range IncP-1 plasmid RK2. J. Mol. Biol. 349, 302-316. doi: 10.1016/j.jmb.2005.03.062

Bouet, J. Y., Ah-Seng, Y., Benmeradi, N., and Lane, D. (2007). Polymerization of SopA partition ATPase: regulation by DNA binding and SopB. Mol. Microbiol. 63, 468-481. doi: 10.1111/j.1365-2958.2006.05537.x

Bouet, J. Y., Surtees, J. A., and Funnell, B. E. (2000). Stoichiometry of P1 plasmid partition complexes. J. Biol. Chem. 275, 8213-8219. doi: 10.1074/jbc.275.11.8213

Breüner, A., Jensen, R. B., Dam, M., Pedersen, S., and Gerdes, K. (1996). The centromere-like parC locus of plasmid R1. Mol. Microbiol. 20, 581-592. doi: 10.1046/j.1365-2958.1996.5351063.x

Carmelo, E., Barillà, D., Golovanov, A. P., Lian, L. Y., Derome, A., and Hayes, F. (2005). The unstructured N-terminal tail of ParG modulates assembly of a quaternary nucleoprotein complex in transcription repression. J. Biol. Chem. 280, 28683-28691. doi: 10.1074/jbc.M501173200

Chen, B. W., Lin, M. H., Chu, C. H., Hsu, C. E., and Sun, Y. J. (2015). Insights into ParB spreading from the complex structure of Spo0J and parS. Proc. Natl. Acad. Sci. U.S.A. 112, 6613-6618. doi: 10.1073/pnas.1421927112

Dam, M., and Gerdes, K. (1994). Partitioning of plasmid R1. Ten direct repeats flanking the parA promoter constitute a centromere-like partition site parC, that expresses incompatibility. J. Mol. Biol. 236, 1289-1298. doi: 10.1016/00222836(94)90058-2

Davis, M. A., and Austin, S. J. (1988). Recognition of the P1 plasmid centromere analog involves binding of the ParB protein and is modified by a specific host factor. $E M B O ~ J .7,1881-1888$.

de la Hoz, A. B., Ayora, S., Sitkiewicz, I., Fernández, S., Pankiewicz, R., Alonso, J. C., et al. (2000). Plasmid copy-number control and better-than-random formation and then to the motor protein through the segrosome.

\section{AUTHOR CONTRIBUTIONS}

MO conceived and wrote this mini-review.

\section{ACKNOWLEDGMENTS}

This work was supported by the Ministerio de Ciencia e Innovación (grant RYC-2011-07900) and by co-funding grant BFU2013-47014-P from the Ministerio de Economía y Competitividad and European Regional Development Fund.

segregation genes of pSM19035 share a common regulator. Proc. Natl. Acad. Sci. U.S.A. 97, 728-733. doi: 10.1073/pnas.97.2.728

de la Hoz, A. B., Pratto, F., Misselwitz, R., Speck, C., Weihofen, W., Welfle, K., et al. (2004). Recognition of DNA by $\omega$ protein from the broad-host range Streptococcus pyogenes plasmid pSM19035: analysis of binding to operator DNA with one to four heptad repeats. Nucleic Acids Res. 32, 3136-3147. doi: 10.1093/nar/gkh633

Delbrück, H., Ziegelin, G., Lanka, E., and Heinemann, U. (2002). An Src homology 3-like domain is responsible for dimerization of the repressor protein KorB encoded by the promiscuous IncP plasmid RP4. J. Biol. Chem. 277, 4191-4198. doi: 10.1074/jbc.M110103200

Dmowski, M., and Kern-Zdanowicz, I. (2016). The Omega (ParB) binding sites together with the RNA polymerase-recognized sequence are essential for centromeric functions of the $P_{\omega}$ region in the partition system of pSM19035. Microbiology 162, 1114-1124. doi: 10.1099/mic.0.000308

Dmowski, M., Sitkiewicz, I., and Ceglowski, P. (2006). Characterization of a novel partition system encoded by the $\delta$ and $\omega$ genes from the streptococcal plasmid pSM19035. J. Bacteriol. 188, 4362-4372. doi: 10.1128/JB.01922-05

Fink, G., and Löwe, J. (2015). Reconstitution of a prokaryotic minus endtracking system using TubRC centromeric complexes and tubulin-like protein TubZ filaments. Proc. Natl. Acad. Sci. U.S.A. 112, E1845-E1850. doi: 10.1073/pnas.1423746112

Funnell, B. E. (1988a). Mini-P1 plasmid partitioning: excess ParB protein destabilizes plasmids containing the centromere parS. J. Bacteriol. 170, 954-960.

Funnell, B. E. (1988b). Participation of Escherichia coli integration host factor in the P1 plasmid partition system. Proc. Natl. Acad. Sci. U.S.A. 85, 6657-6661.

Funnell, B. E., and Gagnier, L. (1993). The P1 plasmid partition complex at parS. II. Analysis of ParB protein binding activity and specificity. J. Biol. Chem. 268, 3616-3624.

Gayathri, P., Fujii, T., Møller-Jensen, J., van den Ent, F., Namba, K., and Löwe, J. (2012). A bipolar spindle of antiparallel ParM filaments drives bacterial plasmid segregation. Science 338, 1334-1337. doi: 10.1126/science.1229091

Ge, Y., Hu, X., Zhao, N., Shi, T., Cai, Q., and Yuan, Z. (2014a). A new tubRZ operon involved in the maintenance of the Bacillus sphaericus mosquitocidal plasmid pBsph. Microbiology 160, 1112-1124. doi: 10.1099/mic.0.075465-0

Ge, Y., Zhao, N., Hu, X., Shi, T., Cai, Q., and Yuan, Z. (2014b). A novel transcriptional activator, tubX, is required for the stability of Bacillus sphaericus mosquitocidal plasmid pBsph. J. Bacteriol. 196, 4304-4314. doi: 10.1128/JB.01855-14

Gerdes, K., Møller-Jensen, J., and Bugge Jensen, R. (2000). Plasmid and chromosome partitioning: surprises from phylogeny. Mol. Microbiol. 37, 455-466. doi: 10.1046/j.1365-2958.2000.01975.x

Golovanov, A. P., Barillà, D., Golovanova, M., Hayes, F., and Lian, L. Y. (2003). ParG, a protein required for active partition of bacterial plasmids, has a dimeric ribbon-helix-helix structure. Mol. Microbiol. 50, 1141-1153. doi: 10.1046/j.1365-2958.2003.03750.x

Gomis-Rüth, F. X., Solá, M., Acebo, P., Párraga, A., Guasch, A., Eritja, R., et al. (1998). The structure of plasmid-encoded transcriptional repressor 
CopG unliganded and bound to its operator. EMBO J. 17, 7404-7415. doi: 10.1093/emboj/17.24.7404

Graham, T. G., Wang, X., Song, D., Etson, C. M., van Oijen, A. M., Rudner, D. Z., et al. (2014). ParB spreading requires DNA bridging. Genes Dev. 28, 1228-1238. doi: $10.1101 / \mathrm{gad} .242206 .114$

Grigoriev, P. S., and Lobocka, M. B. (2001). Determinants of segregational stability of the linear plasmid-prophage N15 of Escherichia coli. Mol. Microbiol. 42, 355-368. doi: 10.1046/j.1365-2958.2001.02632.x

Gruber, S., and Errington, J. (2009). Recruitment of condensin to replication origin regions by $\mathrm{ParB} / \mathrm{SpoOJ}$ promotes chromosome segregation in B. subtilis. Cell 137, 685-696. doi: 10.1016/j.cell.2009.02.035

Guynet, C., and de la Cruz, F. (2011). Plasmid segregation without partition. Mob. Genet. Elements 1, 236-241. doi: 10.4161/mge.1.3.18229

Havey, J. C., Vecchiarelli, A. G., and Funnell, B. E. (2012). ATP-regulated interactions between P1 ParA, ParB and non-specific DNA that are stabilized by the plasmid partition site, parS. Nucleic Acids Res. 40, 801-812. doi: 10.1093/nar/gkr747

Hayes, F., and Barillà, D. (2006). The bacterial segrosome: a dynamic nucleoprotein machine for DNA trafficking and segregation. Nat. Rev. Microbiol. 4, 133-143. doi: 10.1038/nrmicro1342

Hoischen, C., Bussiek, M., Langowski, J., and Diekmann, S. (2008). Escherichia coli low-copy-number plasmid R1 centromere parC forms a U-shaped complex with its binding protein ParR. Nucleic Acids Res. 36, 607-615. doi: 10.1093/nar/gkm672

Huang, L., Yin, P., Zhu, X., Zhang, Y., and Ye, K. (2011). Crystal structure and centromere binding of the plasmid segregation protein ParB from pCXC100. Nucleic Acids Res. 39, 2954-2968. doi: 10.1093/nar/gkq915

Jensen, R. B., Dam, M., and Gerdes, K. (1994). Partitioning of plasmid R1. The parA operon is autoregulated by ParR and its transcription is highly stimulated by a downstream activating element. J. Mol. Biol. 236, 1299-1309. doi: 10.1016/0022-2836(94)90059-0

Khare, D., Ziegelin, G., Lanka, E., and Heinemann, U. (2004). Sequence-specific DNA binding determined by contacts outside the helix-turn-helix motif of the ParB homolog KorB. Nat. Struct. Mol. Biol. 11, 656-663. doi: 10.1038/nsmb773

Kusiak, M., Gapczynska, A., Plochocka, D., Thomas, C. M., and Jagura-Burdzy, G. (2011). Binding and spreading of ParB on DNA determine its biological function in Pseudomonas aeruginosa. J. Bacteriol. 193, 3342-3355. doi: 10.1128/JB.00328-11

Larsen, R. A., Cusumano, C., Fujioka, A., Lim-Fong, G., Patterson, P., and Pogliano, J. (2007). Treadmilling of a prokaryotic tubulin-like protein, TubZ, required for plasmid stability in Bacillus thuringiensis. Genes Dev. 21, 1340-1352. doi: 10.1101/gad.1546107

Leonard, T. A., Butler, P. J., and Löwe, J. (2004). Structural analysis of the chromosome segregation protein Spo0J from Thermus thermophilus. Mol. Microbiol. 53, 419-432. doi: 10.1111/j.1365-2958.2004.04133.x

Lewis, P. J., and Errington, J. (1997). Direct evidence for active segregation of oriC regions of the Bacillus subtilis chromosome and co-localization with the SpoOJ partitioning protein. Mol. Microbiol. 25, 945-954. doi: 10.1111/j.13652958.1997.mmi530.x

Lin, D. C., and Grossman, A. D. (1998). Identification and characterization of a bacterial chromosome partitioning site. Cell 92, 675-685. doi: 10.1016/S00928674(00)81135-6

Livny, J., Yamaichi, Y., and Waldor, M. K. (2007). Distribution of centromere-like parS sites in bacteria: insights from comparative genomics. J. Bacteriol. 189, 8693-8703. doi: 10.1128/JB.01239-07

Martin, K. A., Friedman, S. A., and Austin, S. J. (1987). Partition site of the P1 plasmid. Proc. Natl. Acad. Sci. U.S.A. 84, 8544-8547. doi: 10.1073/pnas.84.23.8544

Minnen, A., Attaiech, L., Thon, M., Gruber, S., and Veening, J. W. (2011). SMC is recruited to oriC by ParB and promotes chromosome segregation in Streptococcus pneumoniae. Mol. Microbiol. 81, 676-688. doi: 10.1111/j.13652958.2011.07722.x

Møller-Jensen, J., Ringgaard, S., Mercogliano, C. P., Gerdes, K., and Löwe, J. (2007). Structural analysis of the ParR/parC plasmid partition complex. EMBO J. 26, 4413-4422. doi: 10.1038/sj.emboj.7601864

Mori, H., Kondo, A., Ohshima, A., Ogura, T., and Hiraga, S. (1986). Structure and function of the F plasmid genes essential for partitioning. J. Mol. Biol. 192, 1-15. doi: 10.1016/0022-2836(86)90459-6
Murayama, K., Orth, P., de la Hoz, A. B., Alonso, J. C., and Saenger, W. (2001). Crystal structure of $\omega$ transcriptional repressor encoded by Streptococcus pyogenes plasmid pSM19035 at 1.5 A resolution. J. Mol. Biol. 314, 789-796. doi: 10.1006/jmbi.2001.5157

Murray, H., Ferreira, H., and Errington, J. (2006). The bacterial chromosome segregation protein Spo0J spreads along DNA from parS nucleation sites. Mol. Microbiol. 61, 1352-1361. doi: 10.1111/j.1365-2958.2006. 05316.x

Ni, L., Xu, W., Kumaraswami, M., and Schumacher, M. A. (2010). Plasmid protein TubR uses a distinct mode of HTH-DNA binding and recruits the prokaryotic tubulin homolog TubZ to effect DNA partition. Proc. Natl. Acad. Sci. U.S.A. 107, 11763-11768. doi: 10.1073/pnas.1003817107

Oliva, M. A., Martin-Galiano, A. J., Sakaguchi, Y., and Andreu, J. M. (2012). Tubulin homolog TubZ in a phage-encoded partition system. Proc. Natl. Acad. Sci. U.S.A. 109, 7711-7716. doi: 10.1073/pnas.11215 46109

Pratto, F., Cicek, A., Weihofen, W. A., Lurz, R., Saenger, W., and Alonso, J. C. (2008). Streptococcus pyogenes pSM19035 requires dynamic assembly of ATPbound ParA and ParB on parS DNA during plasmid segregation. Nucleic Acids Res. 36, 3676-3689. doi: 10.1093/nar/gkn170

Pratto, F., Suzuki, Y., Takeyasu, K., and Alonso, J. C. (2009). Single-molecule analysis of proteinxDNA complexes formed during partition of newly replicated plasmid molecules in Streptococcus pyogenes. J. Biol. Chem. 284, 30298-30306. doi: 10.1074/jbc.M109.035410

Raumann, B. E., Rould, M. A., Pabo, C. O., and Sauer, R. T. (1994). DNA recognition by $\beta$-sheets in the Arc repressor-operator crystal structure. Nature 367, 754-757. doi: 10.1038/367754a0

Ravin, N., and Lane, D. (1999). Partition of the linear plasmid N15: interactions of N15 partition functions with the sop locus of the F plasmid. J. Bacteriol. 181, 6898-6906.

Rice, P. A., Yang, S., Mizuuchi, K., and Nash, H. A. (1996). Crystal structure of an IHF-DNA complex: a protein-induced DNA U-turn. Cell 87, 1295-1306. doi: 10.1016/S0092-8674(00)81824-3

Rodionov, O., Lobocka, M., and Yarmolinsky, M. (1999). Silencing of genes flanking the P1 plasmid centromere. Science 283, 546-549. doi: $10.1126 /$ science. 283.5401 .546

Salje, J., and Löwe, J. (2008). Bacterial actin: architecture of the ParMRC plasmid DNA partitioning complex. EMBO J. 27, 2230-2238. doi: 10.1038/emboj.2008.152

Sanchez, A., Rech, J., Gasc, C., and Bouet, J. Y. (2013). Insight into centromerebinding properties of ParB proteins: a secondary binding motif is essential for bacterial genome maintenance. Nucleic Acids Res. 41, 3094-3103. doi: 10.1093/nar/gkt018

Schumacher, M. A., and Funnell, B. E. (2005). Structures of ParB bound to DNA reveal mechanism of partition complex formation. Nature 438, 516-519. doi: 10.1038/nature 04149

Schumacher, M. A., Glover, T. C., Brzoska, A. J., Jensen, S. O., Dunham, T. D. Skurray, R. A., et al. (2007a). Segrosome structure revealed by a complex of ParR with centromere DNA. Nature 450, 1268-1271. doi: 10.1038/nature 06392

Schumacher, M. A., Mansoor, A., and Funnell, B. E. (2007b). Structure of a four-way bridged ParB-DNA complex provides insight into P1 segrosome assembly. J. Biol. Chem. 282, 10456-10464. doi: 10.1074/jbc.M6106 03200

Schumacher, M. A., Piro, K. M., and Xu, W. (2010). Insight into F plasmid DNA segregation revealed by structures of SopB and SopB-DNA complexes. Nucleic Acids Res. 38, 4514-4526. doi: 10.1093/nar/gkq161

Schumacher, M. A., Ye, Q., Barge, M. T., Zampini, M., Barillà, D., and Hayes, F. (2012). Structural mechanism of ATP-induced polymerization of the partition factor ParF: implications for DNA segregation. J. Biol. Chem. 287, 26146-26154. doi: $10.1074 /$ jbc.M112.373696

Simpson, A. E., Skurray, R. A., and Firth, N. (2003). A single gene on the staphylococcal multiresistance plasmid pSK1 encodes a novel partitioning system. J. Bacteriol. 185, 2143-2152. doi: 10.1128/JB.185.7.2143-21 52.2003

Somers, W. S., and Phillips, S. E. (1992). Crystal structure of the met repressoroperator complex at $2.8 \mathrm{~A}$ resolution reveals DNA recognition by $\beta$-strands. Nature 359, 387-393. doi: 10.1038/359387a0 
Sullivan, N. L., Marquis, K. A., and Rudner, D. Z. (2009). Recruitment of SMC by ParB-parS organizes the origin region and promotes efficient chromosome segregation. Cell 137, 697-707. doi: 10.1016/j.cell.2009.04.044

Surtees, J. A., and Funnell, B. E. (2001). The DNA binding domains of P1 ParB and the architecture of the P1 plasmid partition complex. J. Biol. Chem. 276, 12385-12394. doi: 10.1074/jbc.M009370200

Tang, M., Bideshi, D. K., Park, H. W., and Federici, B. A. (2006). Minireplicon from pBtoxis of Bacillus thuringiensis subsp. israelensis. Appl. Environ. Microbiol. 72, 6948-6954. doi: 10.1128/AEM.00976-06

Weihofen, W. A., Cicek, A., Pratto, F., Alonso, J. C., and Saenger, W. (2006). Structures of $\omega$ repressors bound to direct and inverted DNA repeats explain modulation of transcription. Nucleic Acids Res. 34, 1450-1458. doi: 10.1093/nar/gkl015

Williams, D. R., Macartney, D. P., and Thomas, C. M. (1998). The partitioning activity of the RK2 central control region requires only incC, korB and KorB-binding site $\mathrm{O}(\mathrm{B}) 3$ but other KorB-binding sites form destabilizing complexes in the absence of O(B)3. Microbiology 144(Pt 12), 3369-3378. doi: 10.1099/00221287-144-12-3369

Wu, M., Zampini, M., Bussiek, M., Hoischen, C., Diekmann, S., and Hayes, F. (2011). Segrosome assembly at the pliable parH centromere. Nucleic Acids Res. 39, 5082-5097. doi: 10.1093/nar/gkr115

Yamaichi, Y., and Niki, H. (2000). Active segregation by the Bacillus subtilis partitioning system in Escherichia coli. Proc. Natl. Acad. Sci. U.S.A. 97, 14656-14661. doi: 10.1073/pnas.97.26.14656
Yates, P., Lane, D., and Biek, D. P. (1999). The F plasmid centromere, sopC, is required for full repression of the sopAB operon. J. Mol. Biol. 290, 627-638. doi: 10.1006/jmbi.1999.2909

Yin, P., Li, T. Y., Xie, M. H., Jiang, L., and Zhang, Y. (2006). A Type Ib ParB protein involved in plasmid partitioning in a gram-positive bacterium. J. Bacteriol. 188, 8103-8108. doi: 10.1128/JB.01232-06

Zampini, M., Derome, A., Bailey, S. E., Barillà, D., and Hayes, F. (2009). Recruitment of the ParG segregation protein to different affinity DNA sites. J. Bacteriol. 191, 3832-3841. doi: 10.1128/JB.01630-08

Conflict of Interest Statement: The author declares that the research was conducted in the absence of any commercial or financial relationships that could be construed as a potential conflict of interest.

The handling Editor declared a shared affiliation, though no other collaboration, with the author and states that the process nevertheless met the standards of a fair and objective review.

Copyright (C) 2016 Oliva. This is an open-access article distributed under the terms of the Creative Commons Attribution License (CC BY). The use, distribution or reproduction in other forums is permitted, provided the original author(s) or licensor are credited and that the original publication in this journal is cited, in accordance with accepted academic practice. No use, distribution or reproduction is permitted which does not comply with these terms. 